\title{
An FCA-mediated epigenetic route towards thermal adaptation of autotrophic development in plants
}

\author{
Hyo-Jun Lee ${ }^{1}$,Jun-Ho Ha ${ }^{1}$ E Chung-Mo Park ${ }^{1,2, *}$ \\ ${ }^{1}$ Department of Chemistry, Seoul National University, ${ }^{2}$ Plant Genomics and Breeding Institute, Seoul National University, Seoul 08826, \\ Korea
}

Plants are able to recognize even small changes in surrounding temperatures to optimize their growth and development. At warm temperatures, plants exhibit diverse architectural adjustments, including hypocotyl and petiole elongation, leaf hyponasty, and reduced stomatal density. However, it was previously unknown how such warm temperatures affected the early stages of seedling development. In our recent study, we demonstrated that the RNA-binding protein, FCA, is critical for sustaining chlorophyll biosynthesis during early seedling development, which is a prerequisite for autotrophic transition at warm temperatures. FCA plays a dual role in this thermal response. It inhibits the rapid degradation of protochlorophyllide oxidoreductases (PORs) that mediate chlorophyll biosynthesis. In addition, it induces the expression of POR genes at the chromatin level, which contributes to maintaining functional enzyme levels. Our findings provide molecular basis for the thermal adaptation of chlorophyll biosynthesis during the early stages of seedling development in nature. [BMB Reports 2017; 50(7): 343-344]

Plants synthesize their own energy by fixing carbon from atmospheric $\mathrm{CO}_{2}$, using sun light. Consequently, plants gain an autotrophic competence by inducing chlorophyll biosynthesis and concomitant chloroplast formation during the early stages of seedling development. In this physiological

*Corresponding author. E-mail: cmpark@snu.ac.kr

https://doi.org/10.5483/BMBRep.2017.50.7.070

Received 27 April 2017

Keywords: Autotrophic development, Chlorophyll biosynthesis, FCA, Protochlorophyllide oxidoreductase

Abbreviations: Chlide, chlorophyllide; EIL1, ethylene-insensitive 3-like 1; EIN3, ethylene-insensitive 3; Pchlide, protochlorophyllide; PIF, phytochrome-interacting factor; POR, protochlorophyllide oxidoreductases; ROS, reactive oxygen species

Perspective to: Jun-Ho Ha (2016) Thermo-induced maintenance of photooxidoreductases underlies plant autotrophic development. Developmental Cell, 41, 170-179. doi: 10.1016/j.devcel.2017.03.005 process, protochlorophyllide (pchlide) oxidoreductases (PORs) enzymatically convert pchlide to chlorophyllide (chlide), inducing chlorophyll biosynthesis.

In Arabidopsis, there are three POR enzymes, PORA, PORB, and PORC. Genes encoding POR members are differently regulated in response to changes in light levels. While the expression of PORA gene is reduced to a basal level upon light illumination, the $P O R B$ gene is not significantly influenced by light. Notably, the $P O R C$ gene is induced after seedlings are exposed to light. Light also affects the POR enzyme protein stability. PORA is rapidly destabilized during de-etiolation, possibly by the action of plastid proteases. These regulatory strategies denote that POR abundance is tightly regulated by light during autotrophic development.

Temperature is another environmental cue that profoundly affects morphological and physiological events during plant growth and environmental adaptation. While extreme temperatures induce stress responses in plants, mild fluctuations in the surrounding temperature triggers architectural modifications, as well as physiological adjustments, which are distinct from stress responses. In recent years, the molecular mechanisms underlying plant responses to warm temperature have been extensively studied at the molecular level. It is known that the PHYTOCHROME-INTERACTING FACTOR 4 (PIF4) activates auxin biosynthesis to facilitate cell growth in warm temperatures. In addition, FCA attenuates the function of PIF4 by suppressing its DNA-binding ability to prevent hypocotyl overgrowth in warm temperature conditions.

In our recent study, we have demonstrated that FCA plays a distinct role in regulating autotrophic transition in warm temperatures. While FCA-defective mutants exhibited normal greening when germinated and grown for about 2-3 days at normal temperature prior to exposure to warm temperatures, the $f_{c a}$ mutants exhibited an albino phenotype when they were exposed to warm temperatures from the outset of germination. Since plant autotrophic transition occurs during early seedling development, we hypothesized that FCA is involved in the establishment of autotrophic development in warm temperature conditions.

Our molecular studies indicate that FCA promotes the transcription of $P O R$ genes in warm temperatures by triggering histone acetylation at the POR loci (Fig. 1A). FCA is a 
A

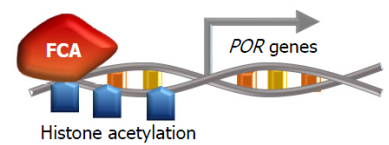

C

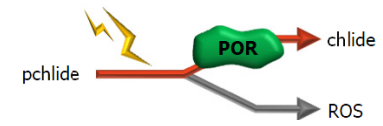

B

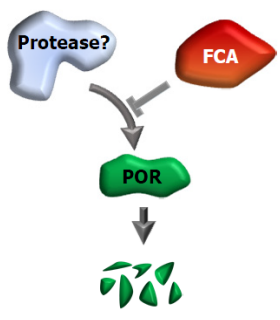

Fig. 1. Schematic diagram of FCA function in sustaining POR abundance at warm temperatures. (A) FCA induces $P O R$ transcription. At warm temperatures, FCA binds to the promoter regions of $P O R$ genes to promote their transcription through histone acetylation. (B) FCA inhibits POR degradation. POR proteins are highly unstable at warm temperatures. FCA stabilizes PORs possibly by suppressing plastid proteases. (C) POR enzymes shifts the chlorophyll-ROS balance toward autotrophic development. POR abundance is maintained by FCA at warm temperatures, which leads to light-induced chlorophyll biosynthesis, while suppressing ROS production.

RNA-binding protein and does not have a DNA-binding domain. It has previously been reported that FCA interacts with PIF4 to aid its binding to the promoter regions of PIF4 target genes. We predict that as-yet unknown transcription factor(s) are involved in the FCA-mediated regulation of $P O R$ expression. PIF1, ETHYLENE-INSENSITIVE 3 (EIN3), and ETHYLENE-INSENSITIVE 3-LIKE 1 (EIL1) are known to directly bind to POR promoters. However, we found that FCA does not interact with these transcription factors directly. For the elucidation of molecular mechanisms underlying FCA function, we must identify upstream transcription factors that are activated in warm temperatures and also interact with FCA. Future work could focus on proteomic analysis with anti-FCA antibodies to investigate this further.

In addition to transcriptional control, FCA also suppresses the destabilization of POR enzymes at warm temperatures (Fig. 1B), although the proteolytic mechanisms underlying POR degradation are not yet understood. Chemical treatment with inhibitors of proteases and 265 proteasome did not rescue the POR degradation in $f_{C a}$ mutants. It has been reported that plastid proteases are responsible for light-mediated POR degradation. It is as such possible that the plastid proteases would mediate the thermo-induced destabilization of POR enzymes. Alternatively, POR enzymes could be degraded through selective autophagy. It is known that denatured protein aggregates are selectively recognized by autophagic machinery for degradation. Investigation of POR protein stability in autophagy-related mutants would be required to confirm this.

The albino phenotype of FCA-deficient mutants is manifested only when developing seedlings are exposed to warm temperatures, suggesting that FCA function is activated by warmth. In accordance with this, it has been reported that FCAmediated inhibition of PIF4 activity is increased with increasing temperatures. Notably, it has recently been reported that the FCA-PIF4 interaction is enhanced when plants are exposed to

warm temperatures. We found that the binding of FCA to the $P O R$ gene promoter was also thermally induced. However, it is largely unknown how FCA is activated in warm temperatures. Gene transcription and protein abundance of FCA are only marginally altered in warm temperature conditions. FCA post-translational modification may explain this observation. Since protein phosphorylation is a representative mechanism for regulating the activity of proteins in most organisms, examination of FCA phosphorylation at warm temperatures could provide critical insight.

In de-etiolating seedlings, POR enzymes rapidly convert pchlide to chlide, as pchlide absorbs light energy. However, light-exposed, but POR-unbound pchlide acts as a photosensitizer that produces reactive oxygen species (ROS) (Fig. 1C). ROS are often considered as toxic chemicals that cause oxidative damage to DNA, protein, and lipids. However, accumulating evidence supports the idea that ROS also play a beneficial role during plant growth and development. In particular, ROS accumulate to high levels during early seedling development, where ROS promote radicle elongation. In our recent study, we found that POR enzymes are highly unstable in warm temperatures during autotrophic development. It is as such possible that plants would reduce POR levels to produce ROS by increasing POR-unbound pchlide levels in warm temperature conditions, in turn facilitating radicle and hypocotyl growth. The seedling growth rate is increased in warm temperatures, to rapidly move leaves and shoot apical meristematic tissue away from the heat-absorbing soil. We propose that this physiological response provides an adaptation strategy, by which developing seedlings utilize ROS as a growth stimulator under warm temperature conditions.

FCA was originally identified as a flowering time regulator. Our recent study identifies a distinct role of FCA, which is associated with autotrophic development. Notably, the role of FCA in autotrophic transition is critical for plant survival, as its mutation causes seedling albinism under warm temperature conditions. It is apparent that FCA mediates chlorophyll biosynthesis, modulating POR protein stability and gene expression. However, details of the regulatory mechanisms remain unknown. Further genetic and biochemical analyses of the FCA-mediated regulation of POR function are required to fully understand autotrophic developmental events that occur in warm temperature conditions.

\section{ACKNOWLEDGEMENTS}

This work was supported by the Leaping Research (NRF2015R1A2A1A05001636) and Global Research Lab (NRF2012K1A1A2055546) Programs, provided by the National Research Foundation of Korea and the Next-Generation BioGreen 21 Program (Plant Molecular Breeding Center No. PJ0111532016), provided by the Rural Development Administration of Korea. 